\title{
An Unusual ENT Presentation of Retinoblastoma: A Diagnostic Dilemma
}

\author{
${ }^{1}$ Tamoghna Jana, ${ }^{2}$ Moushumi Sengupta, ${ }^{3}$ Saumik Das, ${ }^{4}$ Asok K Saha, ${ }^{5}$ Subhasis Saha
}

\begin{abstract}
Retinoblastoma is the most common intraocular tumor of childhood. These tumors, though they respond to treatment, are prone to develop secondary malignancy, recurrence, and metastasis, which may present as sinonasal mass. We are presenting a rare case of metastatic retinoblastoma of sinonasal region in a 3-year-old male child. The mode of presentation and management of the case is presented along with a review of the literature.
\end{abstract}

Keywords: Metastatic, Retinoblastoma, Sinonasal.

How to cite this article: Jana T, Sengupta M, Das S, Saha AK, Saha S. An Unusual ENT Presentation of Retinoblastoma: A Diagnostic Dilemma. Clin Rhinol An Int J 2016;9(3):149-152.

Source of support: Nil

Conflict of interest: None

\section{INTRODUCTION}

Retinoblastoma is the most common intraocular tumor of childhood. This tumor generally has good prognosis following treatment. However, patients who survive retinoblastoma are at risk for developing recurrence, metastasis, and secondary malignancies like soft tissue sarcomas, osteosarcoma, melanoma, brain tumors, and Ewing's sarcoma, most of which may present as a sinonasal mass. Otorhinolaryngologists may be confused by the unusual presentation of retinoblastoma as a sinonasal mass. Furthermore, it is often difficult to diagnose the primary pathology of the second tumor, thus delaying treatment. We have presented a case report of a 3-year-old male patient who had developed a rapidly progressive sinonasal mass within 1 year of treatment of primary retinoblastoma. Diagnosis was reached after taking tissue biopsy, which was followed by review of previous histopathological slides and immunohistochemical studies. Thorough investigations along with review of previous

\footnotetext{
${ }^{1,5}$ Junior Resident, ${ }^{2} \mathrm{RMO}$ and Clinical Tutor, ${ }^{3,4}$ Associate Professor

${ }^{1-5}$ Department of Otorhinolaryngology, Medical College and Hospital, Kolkata, West Bengal, India
}

Corresponding Author: Moushumi Sengupta, RMO and Clinical Tutor, Department of Otorhinolaryngology, Medical College and Hospital, Kolkata, West Bengal, India, Phone: +919433360559, e-mail: drmou_sen@rediffmail.com histopathological slides and immunohistochemical study are therefore an essential tool to rule out any diagnostic dilemma.

\section{CASE REPORT}

A 3-year-old male child presented to the ear, nose, and throat outpatient department of the Medical College and Hospital in April 2013 with a painful, tender swelling of the right side of face, which developed 3 weeks earlier and was rapidly increasing in size. The patient had a history of enucleation of the left eye in June 2012. Histopathological examination confirmed it as primary retinoblastoma with involvement of the choroid and sclera for which the patient received postoperative radiotherapy by 21 fraction, which was completed in September 2012.

On clinical examination, the swelling was firm to hard in consistency, tender, irregular surfaced, fixed to the overlying skin, and measuring about $7 \times 5 \mathrm{~cm}$ in dimensions. It was extending medially pushing the external nasal pyramid to the opposite side, laterally up to the zygoma, superiorly up to inferior orbital margin pushing the eyeball upward, and inferiorly up to the level of upper lip (Fig. 1). The hard and soft palate on the right side was pushed downward, and there was loosening of multiple teeth with dental malocclusion. On ophthalmological evaluation of right eye, visual acuity was found to be normal with absent white eye reflex. Funduscopic examination was also normal, thus ruling out the possibility of retinoblastoma of the right eye.

Contrast-enhanced computed tomography of paranasal sinuses revealed an expansile heterogeneous soft tissue lesion occupying both the maxillary antra. There was erosion of anterolateral wall of right maxilla with invasion of soft tissue of right cheek and also erosion of medial wall with extension into the nasal cavity on right side and pushing the posterolateral wall posteriorly. There was also a heterogeneous opacity in left maxillary antrum eroding the anterolateral and medial wall. The palatine process of maxilla was eroded with bony remodeling of anterior wall of right maxilla involving both the orbits, with erosion of floor of the orbits (Figs 2 and 3). Radiological features were suggestive of malignant round cell tumor of sinonasal region.

One week later, the child developed a tender swelling over proximal $1 / 3$ rd of right forearm. On radiological 
examination, periosteal reaction with sun ray pattern was seen over the right ulna (Fig. 4).

Nasal endoscopy did not reveal any abnormality in the nasal cavities, but the right lateral nasal wall was found to be pushed toward the septum almost touching it. A punch biopsy was taken from the soft tissue overlying the anterior wall of right maxilla through sublabial approach. Histopathological examination showed sheets of round or oval cells with large hyperchromatic nuclei, suggestive of malignant round cell tumor (Fig. 5). On immunohistochemistry, tumor cells were found to be positive for synaptophysin and chromogranin A, thus suggesting a diagnosis of either retinoblastoma or olfactory neuroblastoma. The tumor was found to be negative for cytokeratin, epithelial membrane

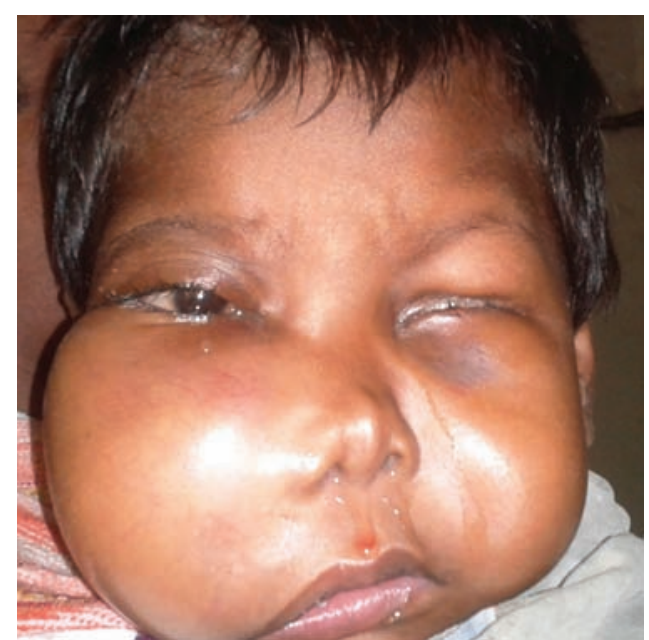

Fig. 1: Clinical photograph of patient showing swelling on the right side of face

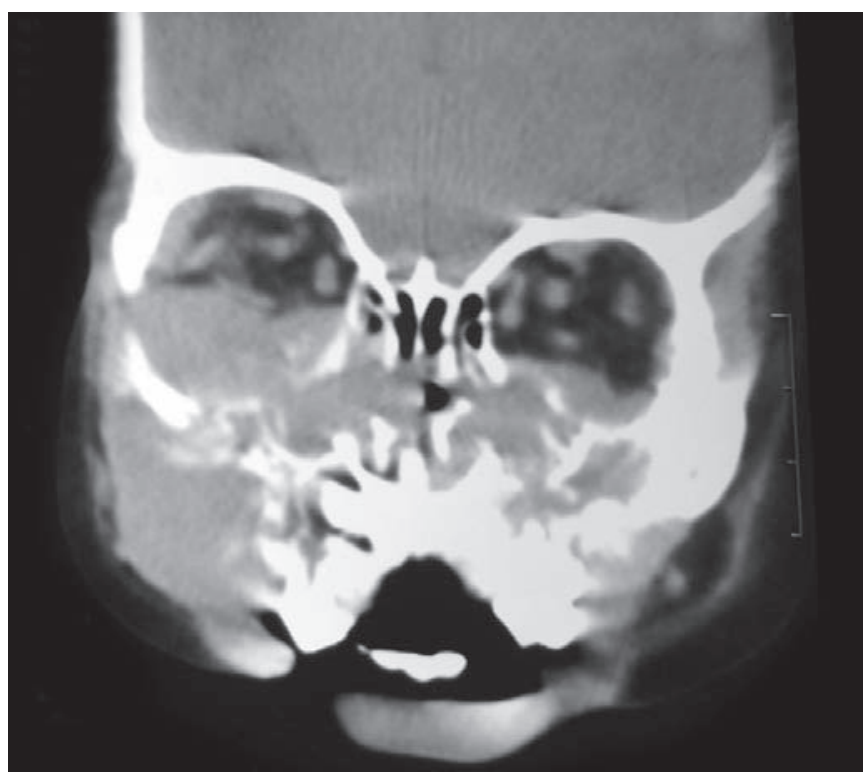

Fig. 3: Contrast-enhanced computed tomography of paranasal sinuses (coronal section) showing involvement of right orbit antigen, MIC-2, leukocyte common antigen, desmin, and myogenin, thus ruling out carcinoma, epithelial malignancy, Ewing's sarcoma, non-Hodgkin's lymphoma (NHL), myeloid sarcoma, and rhabdomyosarcoma respectively.

But, as in the computed tomography (CT) scan, olfactory region was free from tumor, and as the age of the child was also against the diagnosis of olfactory neuroblastoma, a review of all the previous histopathological slides including that of the primary retinoblastoma was done, which was followed by immunohistochemistry. The final histopathological interpretation was that of a distal recurrence of retinoblastoma.

The child was started on standard chemotherapy protocol for retinoblastoma on four weekly cycle in May

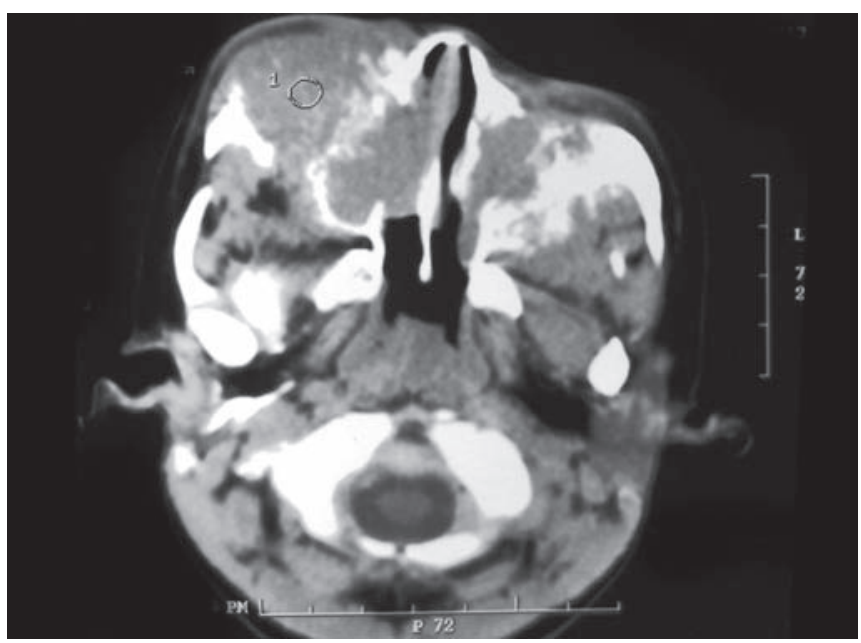

Fig. 2: Contrast-enhanced computed tomography of paranasa sinuses (axial section) showing an expansile heterogeneous mass occupying both the maxillary antra with bone erosion and soft tissue invasion of right cheek

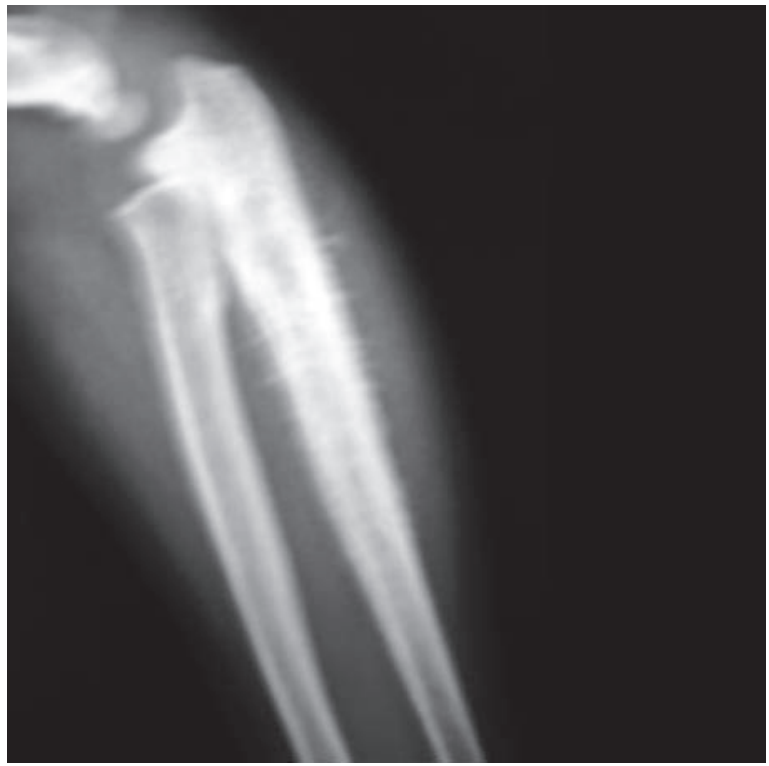

Fig. 4: Radiological picture of right forearm showing soft tissue swelling with "sunray pattern" 


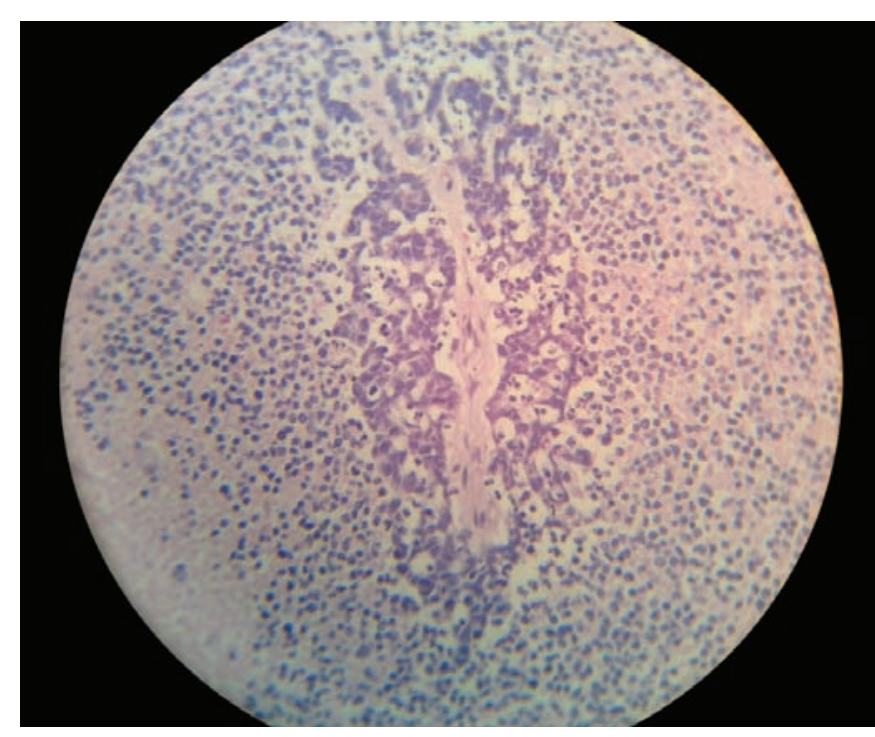

Fig. 5: Hematoxylin-eosin stain of biopsy sample showing small, blue round cells

2013, comprising vincristine $1.5 \mathrm{mg} / \mathrm{m}^{2}$ on day 1 , carboplatin $560 \mathrm{mg} / \mathrm{m}^{2}$ on day 1 , and etoposide $150 \mathrm{mg} / \mathrm{m}^{2}$ on days 1 and 2.

The child significantly improved after receiving four cycles of chemotherapy, the swelling over right side of the face decreased considerably, proptosis of right eye and the tender swelling over the forearm subsided, thus improving the general well-being of the patient (Fig. 6).

\section{DISCUSSION}

Nonepithelial neoplasms constitute the majority of sinonasal tumors in children. Among these, rhabdomyosarcoma or undifferentiated sarcoma and NHLs account for the majority of cases. Less frequently, Ewing's sarcoma or primitive neuroendocrine tumors can present in this location. ${ }^{1}$ These tumors have also been described as secondary malignancies following treatment of retinoblastoma and other neoplasms. Esthesioneuroblastoma is a rare sinonasal tumor. Other less common sinonasal tumors presenting in children include hemangioma and hemangiopericytoma, fibroma and fibrosarcoma, malignant fibrous histiocytoma, and desmoid fibromatosis.

Retinoblastoma, though not familiar to otorhinolaryngologists, is the most common intraocular tumor of childhood.

Retinoblastoma presents with cumulative lifetime incidence rate of 18,000 to 30,000 live births worldwide. ${ }^{2}$ A higher incidence is noted in developing countries, which may be implicated to lower socioeconomic status and the presence of human papilloma virus sequences in the retinoblastoma tissue. ${ }^{3}$

It is a highly malignant, primary retinal tumor arising from neuroectodermal cells that are destined to become retinal photoreceptors. A mutation in RB-1

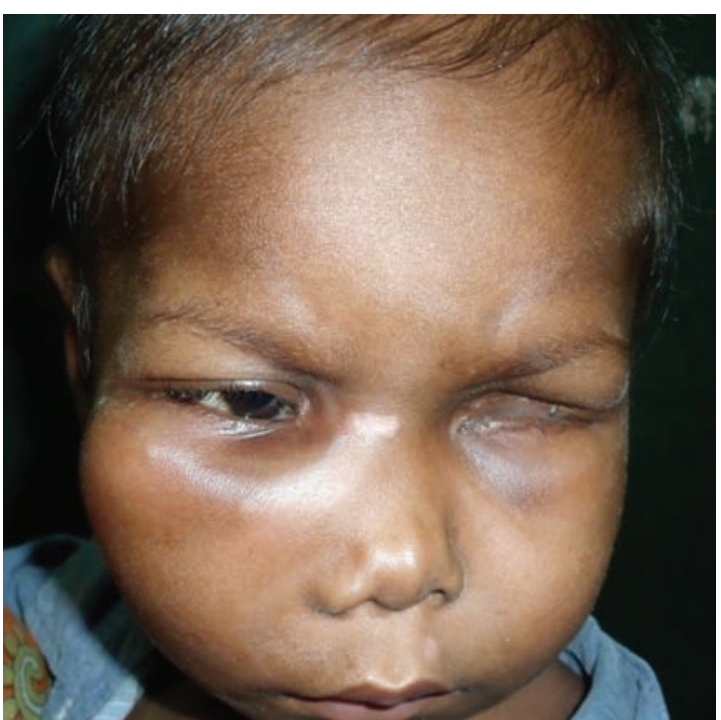

Fig. 6: Clinical photograph of patient following treatment showing reduction in the size of the swelling

gene in chromosome 13q14 is responsible. ${ }^{4}$ Survival rate in the developed world is $99 \%$ with the advent of newgeneration chemoradiation - highest survival rate of all pediatric cancers. ${ }^{5}$

The prognosis for most children diagnosed with retinoblastoma is good, with a 5-year survival rate of more than $90 \%$. Complications include local recurrence, metastases, and the development of a second primary tumor.

Recurrence in retinoblastoma is of two types - local when it occurs in the same orbit and distal when it occurs outside the orbit. In our patient, it was found to be a case of distal metastasis.

There are mainly five routes of metastasis: (1) cerebrospinal fluid; (2) bloodstream; (3) orbit to orbit by optic nerve; (4) contiguity; and (5) lymphatic. In our case, it most probably spread by hematogeneous route.

The common site of metastasis in case of retinoblastoma is the intracranial region accounting for about $50 \%$ of cases. Bone marrow and bone are respectively 2 nd and 3rd preferred secondary sites. Bone metastasis occurs frequently to the skull and long bones via the vascular route, ${ }^{4}$ which is usually seen to occur within 2 years of initial diagnosis. In our case, metastasis was seen in opposite maxilla and zygoma, which presented to us as a sinonasal mass. Skeletal metastasis to the opposite maxilla and zygoma in a case of retinoblastoma is a rare presentation and has not been previously reported. Also, though involvement of long bones is common, forearm involvement is particularly rare, which was seen in our case. Involvement of multiple bones would point the diagnosis toward metastases rather than multifocal second primary. Imaging modalities available for skeletal metastatic workup include $\mathrm{X}$-ray, $\mathrm{CT}$, magnetic resonance 
imaging, and functional imaging modalities like bone scan where available.

Survivors of the hereditary form of retinoblastoma have a much higher risk for developing other types of cancer throughout their lives. This is because each cell in the body has an abnormal RB1 tumor suppressor gene, which would normally help stop some of these cancers from forming. Risk of second malignancy after retinoblastoma is as high as $20 \%$ at 10 years; in irradiated cases, it may go up to $35 \%$. Most of these cancers are treatable if detected early, which is why it is very important that these patients are followed closely throughout life. The entire body must be carefully examined to avoid missing these second cancers. ${ }^{6}$

Second malignancies that may occur in these patients are osteosarcoma, leiomyosarcoma, spindle cell sarcoma, malignant fibrous histiocytoma, rhabdomyosarcoma, angiosarcoma, Ewing sarcoma, melanoma, brain tumors, lung cancer, lymphoma, and breast cancer. These typically develop after a latent period of 8 to 15 years. Many of these malignancies may be manifested as a sinonasal mass, which comes under the domain of otorhinolaryngology.

\section{CONCLUSION}

Retinoblastoma though has a good prognosis following timely treatment, may be complicated by the development of recurrence, metastasis, and second malignancies. Otorhinolaryngologists are often confused regarding the diagnosis of the second pathology when it presents as a sinonasal mass. Moreover, it is difficult to distinguish second malignancy when occurring early from recurrent retinoblastoma (especially distal), but is worth determining, because treatment and the prognosis may differ considerably. Histopathological finding and immunohistochemical studies, thus, are an essential tool to reach a diagnosis with early intervention.

\section{REFERENCES}

1. Fact Sheet: Pediatric Head and Neck Tumors. American Academy of Otolaryngology - Head and Neck Surgery.

2. Abramson DH, Schefler AC. Update on retinoblastoma. Retina 2004 Dec;24(6):828-848.

3. Orjuela M, Castaneda VP, Ridaura C, Lecona E, Leal C, Abramson DH, Orlow I, Gerald W, Cordon-Cardo C. Presence of human papilloma virus in tumor tissue from children with retinoblastoma: an alternative mechanism for tumor development. Clin Cancer Res 2000 Oct; 6(10):4010-4016.

4. Taguchi A,Suei Y, Ogawa I,Naito K, Nagasaki T, LeeK, Fujita M, Tanimoto K. Metastatic retinoblastoma of the maxilla and mandible. Dentomaxillofacial Radiol 2005 Mar;34(2):126-131.

5. Lin $\mathrm{P}, \mathrm{O}^{\prime}$ Brien JM. Frontiers in the management of retinoblastoma. Am J Ophthalmol 2009;148(2):192-198.

6. Availablefrom:http://www.cancer.org/cancer/retinoblastoma/ detailedguide/retinoblastoma-after-second-cancers 